\title{
Correction to: Cardiac Differentiation of Mesenchymal Stem Cells: Impact of Biological and Chemical Inducers
}

\author{
Saravanan Ramesh ${ }^{1} \cdot$ Kavitha Govarthanan ${ }^{2} \cdot$ Serge Ostrovidov ${ }^{3} \cdot$ Haiguang Zhang $^{4} \cdot$ Qingxi Hu${ }^{4}$. \\ Gulden Camci-Unal ${ }^{5} \cdot$ Rama S. Verma $^{2} \cdot$ Murugan Ramalingam $^{6}$ (B)
}

Published online: 25 May 2021

(c) Springer Science+Business Media, LLC, part of Springer Nature 2021

\section{Correction to: Stem Cell Reviews and Reports https://doi.org/10.1007/s12015-021-10165-3}

The original version of this article unfortunately contained a mistake. The affiliation of the authors, Kavitha Govarthanan and Rama S Verma, was incorrect. The correct information is given below.

The original article has been corrected.

The original article can be found online at https://doi.org/10.1007/ s12015-021-10165-3.

Murugan Ramalingam

rmurug2000@gmail.com

1 School of Bio Sciences and Technology, Vellore Institute of Technology, Vellore 632014, India

2 Stem Cell and Molecular Biology Lab, Bhupat and Jyoti Mehta School of Biosciences, Department of Biotechnology, Indian Institute of Technology Madras, Chennai 600036, India

3 Center for Minimally Invasive Therapeutics (C-MIT), Department of Radiological Sciences, University of California, Los Angeles, CA 90095, USA

4 Rapid Manufacturing Engineering Center, Shanghai University, Shanghai 200444, China

5 Department of Chemical Engineering, University of Massachusetts Lowell, One University Ave, Lowell, MA 01854, USA

6 Biomaterials and Organ Engineering Group, Centre for Biomaterials, Cellular and, Molecular Theranostics, School of Mechanical Engineering, Vellore Institute of Technology, Vellore 632014, India
Publisher's Note Springer Nature remains neutral with regard to jurisdictional claims in published maps and institutional affiliations. 